\title{
Grain growth in U-7Mo alloy: a combined first-principles and phase field study
}

\author{
Zhi-Gang Mei*, Linyun Liang, Yeon Soo Kim, Tom Wiencek, Edward O’Hare, \\ Abdellatif M. Yacout, Gerard Hofman, Mihai Anitescu \\ Argonne National Laboratory, 9700 South Cass Avenue, Argonne, IL 60439 - USA
}

\begin{abstract}
Grain size is an important factor in controlling the swelling behavior in irradiated U-Mo dispersion fuels. Increasing the grain size in U-Mo fuel particles by heat treatment is believed to delay the fuel swelling at high fission density. In this work, a multiscale simulation approach combining first-principles calculation and phase field modeling is used to investigate the grain growth behavior in U-7Mo alloy. The density functional theory based first-principles calculations were used to predict the material properties of U-7Mo alloy. The obtained grain boundary energies were then adopted as an input parameter for mesoscale phase field simulations. The effects of annealing temperature, annealing time and initial grain structures of fuel particles on the grain growth in U-7Mo alloy were examined. The predicted grain growth rate was compared well with the empirical correlation derived from experiments.
\end{abstract}

* Corresponding author. Tel.: +1-630-252-2318.

E-mail address: zmei@anl.gov (Z.G. Mei) 


\section{Introduction}

U-Mo alloys are one of the most promising fuels for the future high performance research and test reactors due to its high uranium loading density and stable irradiation behavior [1]. Fission induced swelling in U-Mo fuels is generally stable and predictable. A major contribution to the swelling comes from the yield of fission gases. Because of the extremely low solubility, fission gas atoms exist either in host lattice sites, or inside gas bubbles. In research reactors the operation temperature is usually low $\left(<200{ }^{\circ} \mathrm{C}\right)$. Therefore, the formation and growth of fission gas bubbles are attributed mainly to the fission-enhanced diffusion [2]. At low burnups, fuel swelling by forming fission gas bubbles shows a stable linear dependence of fission density. Because of the high burnups pursued in U-Mo fuels, a phenomenon called recrystallization or grain subdivision takes place, which expedites the formation and growth of gas bubbles. Acceleration in fuel swelling at high burnups is closely related to this phenomenon. Recrystallization has been found to start at existing grain boundaries [3, 4]. Hence increasing the grain size and reducing the grain boundary area by the thermal treatment of U-Mo alloy can effectively delay the recrystallization [5]. In addition to increasing the grain size, heating at high temperature is expected to anneal the defects introduced during powder production, further delaying the recrystallization.

Several heat treatment approaches have been used for atomized U-Mo alloy powders. In the late 1990s, the performance of heat-treated U-10 wt.\% Mo powders (simplified as U-10Mo, and similarly for U-7Mo) d was investigated in the RERTR-3 test irradiated in the ATR [6]. The atomized powders were heat treated at $800{ }^{\circ} \mathrm{C}$ for 100 hours before hot-rolling of fuel plates. The temperature was selected to preserve the $\gamma$ - 
phase of U-10Mo alloy. Considerable grain growth was observed after the heat treatment. Recently, Kim et al. heat treated atomized U-7Mo at $1000{ }^{\circ} \mathrm{C}$ for an hour in a vacuum furnace in order to apply a uranium nitride (UN) coating layer [5], in which the UN coating was applied to prevent the interaction between $\mathrm{U}-7 \mathrm{Mo}$ fuel particle and $\mathrm{Al}$ matrix. After heating, the grain size in U-7Mo powders increases from $6 \mu \mathrm{m}$ to about $20 \mu \mathrm{m}$. Recently we also treated U-7Mo powders at $900{ }^{\circ} \mathrm{C}$ for one hour in quartz glass [7]. The grain size after heat treatment was found to be in the range of $10 \mu \mathrm{m}$ and $30 \mu \mathrm{m}$. In order to obtain the desired grain size and grain size distribution, more heat treatment experiments should be conducted with different annealing time and temperatures, which are important for understanding the growth kinetics of the U-Mo alloys.

Mesoscale simulation methods, such as the phase field modeling, also have been extensively used to study the temporal and spatial evolution of microstructures in various materials $[8,9]$. The kinetic and topological behaviors of grain growth in polycrystalline materials can be well predicted by phase field methods, which also compare well with analytical models. Because of the scarcity of the fundamental thermophysical properties of U-Mo alloys, however, few phase field studies of the microstructure evolution in UMo alloys have been conducted, and most of these have relied on unphysical parameters.

The $\gamma$-phase of the U-Mo alloy used as nuclear fuel for research reactors is a solid solution system with a body-centered cubic (bcc) structure, which is stable only in the high temperature region. One of the common difficulties in atomistic simulation of solid solution systems is to mimic the random structure in a finite supercell. In first-principles calculations for solid solution systems, several approximations are used, such as the virtual crystal approximation (VCA) method proposed by Bellaiche and Vanderbilt [10]. 
In VCA, pseudopotentials are generated as the weighted average of the pseudopotential for each atomic species. However, the method is not suitable for alloys containing atoms of vastly different sizes. Recently, the special quasirandom structure (SQS) model proposed by Zunger et al [11] has gained more popularity for analyzing the properties of solid solution systems. In this model, the randomness of atom distribution is introduced by emulating the correlation functions of an infinite random alloy within a finite supercell. The SQS models have been successfully applied to a number of alloy systems, such as U$\mathrm{Zr}$ [12] and U-Mo [13]. For the phase field simulation of grain growth, the most important input parameters are the grain boundary properties, such as grain boundary energy and grain boundary mobility. To the best of our knowledge, no density functional theory (DFT) or molecular dynamics study of the grain boundary properties in $\gamma$ U-Mo alloy have been carried out.

To this end, we investigated the grain growth in the $\gamma$ U-7Mo alloy using a combined first-principles and phase field simulation approach. DFT-based first-principles calculations were utilized to predict the fundamental material properties of U-7Mo alloy. Using the predicted properties and applying the phase field method, we studied the effects of annealing temperature, annealing time and initial grain structure on the grain growth in U-7Mo alloy. The obtained grain growth rate was compared with the empirical correlation derived from experiment.

In Section 2, we discuss the methodology and computational details of the DFT calculations and phase field modeling. In Section 3 the results are presented and compared with experimental data. A summary of this work is given in Section 4. 


\section{Computational methodology}

We first describe the computational details of DFT calculations and then discuss the formulation of the phase field model.

\subsection{DFT calculations}

In this study, the atomic structures of U-Mo alloys were modeled by the SQS method using the Alloy Theoretic Automated Toolkit (ATAT) code [14, 15]. The SQS models of the bulk U-Mo alloy phase were constructed from a $4 \times 4 \times 4$ bcc supercell with 128 atoms. As an example, Figure 1 shows the atomic structure of U-7Mo alloy using SQS. To perform DFT calculations, we used the projector augmented wave method (PAW) [16] as implemented in the Vienna ab initio simulation package (VASP) $[17,18]$. The exchange-correlation functional was described by the generalized gradient approximation (GGA) parameterized by Perdew Burke and Ernzerhof [19]. The $6 s^{2} 6 p^{6} 5 f^{2} 7 s^{2}$ and $4 s^{2} 4 p^{6} 4 d^{5} s^{1}$ electrons were treated as valence electrons for $\mathrm{U}$ and Mo, respectively. The crystal structures were fully relaxed with respect to cell shape, cell volume, and atomic positions. In all cases the convergence criteria for the total energy and forces were set to $10^{-7} \mathrm{eV} /$ atom and $10^{-2} \mathrm{eV} / \AA$. During the total energy calculations, the cut-off energy for the plane-wave expansion was set as $400 \mathrm{eV}$. Brillouin zone integrations were performed by using a set of $2 \times 2 \times 2 k$-points. Tests were made by using $3 \times 3 \times 3 k$-points in the case of $\mathrm{U}-7 \mathrm{Mo}$ as an example, and the final errors of the total energy difference were found to be less than $10^{-3} \mathrm{eV}$.

Two symmetric tilt grain boundaries (STGBs), $\Sigma 3(111)[110]$ and $\Sigma 5(310)[001]$, were studied in this work; these are frequently observed in bcc metal systems [20],. The 
standard method was used to construct the coincidence site lattice grain boundaries. The atomic structures for the STGBs were generated by GBstudio [21]. For the $\Sigma 3(111)$ GB, one grain of bcc metal was rotated against the other grain around the common [110] axis by about $70.53^{\circ}$, and the (111) plane was set as the GB plane. The $\Sigma 3(111)[110]$ GB was constructed by using a orthorhombic supercell with 48 atoms as shown in Fig. 2 (a). The unit vectors of the 48 -atom based orthorhombic cell are $(1,1,2) a_{0},(1,1,0) a_{0}$, and $(4,4$, 4) $a_{0}$, with $a_{0}$ the lattice constant of the bcc metal, such as Mo. Similarly, the $\Sigma 5(310)$ [001] GB was constructed by rotating one grain by about $36.9^{\circ}$, and the (310) plane is set as the GB plane. The $\Sigma 5(310)$ GB was constructed by using a orthorhombic supercell with 40 atoms as shown in Fig. 2 (b), with the unit vectors of the supercell as $(-1,3,0) a_{0},(0,0$, 1) $a_{0}$, and $(6,2,0) a_{0}$. The $\Sigma 3(111)[110]$ and $\Sigma 5(310)[001]$ GBs for the U-7Mo alloy system were created by applying the SQS model to the GB structures generated for the

pure metal system, as shown in Fig. 3. Brillouin zone integrations were performed by using $k$-point meshes of $7 \times 12 \times 3$ and $5 \times 8 \times 3$ for the supercells of the $\Sigma 3(111)[110$ ] and $\Sigma 5(310)[001]$ GBs, respectively. Other settings for the DFT calculations are similar to those for the bulk phases.

\subsection{Phase field model formulation}

In the phase field model, a large number of phase field variables $\eta_{i}(r)$ were used to distinguish the different orientations of grains, where the subscript $i$ represents the number of grains. These variables are continuous functions of spatial coordinates and time. Within grains, the absolute value for $\eta_{i}$ is one, while all other $\eta_{j}(j \neq i)$ is zero. The value of $\eta_{i}$ varies continuously from one to zero across the grain boundary. The total 
free energy of the interested system can be represented in a Ginsburg-Landau form as [22, 23]

$$
F=\int\left[f_{0}\left(\eta_{1}, \eta_{2}, \ldots, \eta_{q}\right)+\frac{1}{2} \kappa \sum_{i} \nabla^{2} \eta_{i}(r)\right] d^{3} r
$$

where $f_{0}$ is the local free energy density of the system, the second term is the gradient energy term and $\kappa$ is its gradient coefficient.

For modeling the grain growth in a pure system, the local free energy density function is constructed as $[22,23]$

$$
f_{0}\left(\eta_{1}, \eta_{2}, \ldots, \eta_{q}\right)=\sum_{i=1}^{q}\left(-\frac{A}{2} \eta_{i}^{2}+\frac{B}{4} \eta_{i}^{4}\right)+C \sum_{i=1}^{q} \sum_{j \neq i}^{q} \eta_{i}^{2} \eta_{j}^{2}+\frac{1}{4}
$$

where $A, B$, and $C$ are positive constants, and $q$ represents the total number of grain orientations in a grain structure. If we integrate the free energy, it is entirely related to the grain boundary properties. Thus, the expansion coefficients of the free energy can be determined from the grain boundary properties of U-7Mo alloys. [24]

The spatial and temporal evolutions of grain parameters follow the Allen-Cahn equation [9],

$$
\frac{\partial \eta_{i}}{\partial t}=-L \frac{\delta F}{\delta \eta_{i}}, i=1,2, \ldots, q
$$

where $L$ is the kinetic coefficient of grain boundary movement.

To take into account the spherical shape of the U-7Mo alloy particle, we introduce a domain parameter to distinguish the U-7Mo particle and the Al matrix as shown in Fig. 4. A domain parameter $\phi$ is introduced to describe the U-7Mo phase with $\phi=1$ and the Al matrix phase with $\phi=0$ as depicted in the figure, where $\phi$ is a continuous variable and 
smoothly change from 0 to 1 across the interface between the U-7Mo particle and $\mathrm{Al}$ matrix. Since the particle is fixed in our simulation, $\phi$ does not evolve with time. The kinetic coefficient $L_{0}$ depends on the domain parameter as [25], $L_{0}(\phi)=L_{\alpha} h(\phi)+$ $L_{\beta}(1-h(\phi))$. Here $L_{\alpha}$ is the kinetic coefficient for the U-7Mo particle, and $L_{\beta}$ is a very small value to ensure that no grain boundary movement occurs outside the U-7Mo particle, and $h(\phi)$ is an interpolation function having the form $h(\phi)=\phi^{3}\left(6 \phi^{2}-15 \phi+\right.$ 10), which is plotted in Fig. 4(b).

The temperature effect can be considered in the kinetic coefficient $L$ according to the Arrhenius formula as [26]

$$
L(\phi)=L_{0}(\phi) e^{-\frac{Q}{k_{B} T}}
$$

where $k_{B}$ is the Boltzmann's constant, $T$ is the temperature, and $Q$ is the activation energy of grain boundary diffusion.

In order to quantitatively simulate the U-7Mo alloy based on this model, the grain boundary energy, grain boundary mobility, and activation energy have to be determined by atomic calculations or experiments. In this work, the grain boundary energy is predicted by DFT method as outline earlier. Based on this information, the expansion coefficients of chemical free energy and the gradient coefficients can be determined. Due to the difficulty of calculating the grain boundary mobility, this value will be calibrated based on the experimental measurement of grain size with different annealing times. The details of deriving these parameters are provided in Sec. 3. 
The phase field model was implemented in an in-house simulation code and the semi-implicit FFTW numerical method was employed to solve the Allen-Cahn equations [27]. Periodic boundary conditions were imposed on the simulation domain. The time step for the evolution was $t=3.0 \mathrm{~s}$, and the spacing was $\Delta \mathrm{x}=\Delta \mathrm{y}=1.0 \mu \mathrm{m}$. A model size of $200 \mu \mathrm{m} \times 200 \mu \mathrm{m}$ and a U-7Mo circular plate size of $180 \mu \mathrm{m}$ were used in the simulations.

\section{Results and discussion}

We discuss in this section the materials properties and grain growth results from the DFT and phase field modeling studies, respectively.

\subsection{Material properties of U-Mo alloys by DFT}

Consistent with previous investigations of $U$ metal, our study shows that the bcc $\gamma$-phase of $U$ is stable only at high temperatures. The calculated total energy of the $\gamma$ - $U$ phase is about $0.27 \mathrm{eV}$ higher than that of the low-temperature $\alpha-\mathrm{U}$ phase. Elements, such as Mo and $\mathrm{Zr}$, are therefore used to stabilize the $\gamma$-U phase to lower temperatures. To evaluate the thermodynamic stability of the $\gamma$ U-Mo alloy, we estimated the enthalpy of formation of the $\gamma$ U-Mo alloy phase. With bcc Mo and $\gamma$-U as the reference states for

elements Mo and $\mathrm{U}$, the calculated enthalpy of formation shows that $\gamma$ U-7Mo alloy is thermodynamically stable at high temperature above $600{ }^{\circ} \mathrm{C}$ while it is unstable at room temperature with respect to bcc Mo and $\alpha-\mathrm{U}$ (more details will be presented elsewhere). Therefore the $\gamma$ U-7Mo phase observed at room temperature must be a metastable phase. 
To further examine the mechanical stability of the $\gamma$ U-Mo alloys, we investigated the elastic properties of the $\gamma$ U-Mo phases. Single-crystal elastic constants of a solid phase can be calculated by the stress-strain method [28]. In this work, the single-crystal elastic constants are determined based on the applied strains of \pm 0.01 . From the predicted elastic constants, the polycrystalline properties including bulk modulus $(B)$, shear modulus $(G)$, Young's modulus $(E)$ and Poisson's ration $(v)$ can be determined by the Voight-Reuss-Hill approach [29]. Table I summarizes the predicted single-crystal elastic constants and polycrystalline properties of bcc Mo, $\gamma$-U and U-7Mo phases. In these three systems, only bcc Mo has been previously measured for its elastic properties. The currently predicted elastic constants and polycrystalline moduli of bcc Mo agree perfectly with the experimental data by Dickinson and Armstrong [30], with an average error less than 2\%. In comparison with bcc Mo, the predicted elastic constants of $\gamma-\mathrm{U}$ are considerable smaller, especially the bulk modulus, indicating its weaker bonding than that in bec Mo.

The shear constant $C^{\prime}=\left(C_{11}-C_{12}\right) / 2$ of $\gamma-\mathrm{U}$ is predicted to be negative. The negative shear constant of the $\gamma-\mathrm{U}$ phase indicates its mechanical instability, which is in agreement with the previous study by Beeler et al [31]. So far, because of the mechanically unstable nature at room temperature, no experimental or theoretical study of the elastic properties of $\gamma$ U-Mo alloys has been carried out. Predicting the elastic properties of alloy phases by DFT is theoretically challenging. The SQS method in general breaks the point group symmetry. The configurational disorder usually results in a triclinic lattice for a finite supercell. For the bcc $\gamma$ U-Mo alloy phases, the SQS approach results in elastic tensors with 21 nonvanishing elements. Theoretically, there 
should be only three independent elastic constants $C_{11}, C_{12}$, and $C_{44}$ for cubic systems. According to Tasnadi et al [32], the closest cubic elastic constants can be obtained by simple averaging,

$$
\bar{C}_{11}=\frac{C_{11}+C_{22}+C_{33}}{3}, \bar{C}_{12}=\frac{C_{12}+C_{13}+C_{23}}{3}, \text { and } \bar{C}_{44}=\frac{C_{44}+C_{55}+C_{66}}{3} \text {. }
$$

The cubic-averaged elastic constants of the $\gamma$ U-7Mo alloy together with its polycrystalline properties are shown in Table I. Upon alloying Mo in $\gamma-U$, the shear constant $C^{\prime}$ of $\gamma$ U-7Mo alloy becomes positive, indicating that the isotropic $\gamma$ phase of $U$ can be mechanically stabilized by alloying Mo.

Based on the studies of the bulk phase, we further investigated the grain boundary properties in $\gamma$ U-Mo alloys. In this work, we focus on only two representative STGBs in the bcc $\gamma$ U-7Mo alloy: $\Sigma 3(111)[110]$ and $\Sigma 5(310)[001]$ GBs. We define the grain boundary energy $\left(\gamma_{\mathrm{GB}}\right)$ as

$$
\gamma_{G B}=\frac{E_{G B}-E_{S C}}{2 A}
$$

where $E_{\mathrm{GB}}$ and $E_{\mathrm{SC}}$ are the total energies of the GB supercell and its corresponding single crystal, respectively. The latter is obtained by the bulk supercell with periodicity similar to that of the GB supercell. $A$ is the area of the GB plane, and the expression is divided by 2 because of the two interfaces in the supercell. For completeness, we also study the GBs of bec Mo and $\gamma$-U. Figure 2 shows the created atomic structures of the $\Sigma 3(111)[110]$ and $\Sigma 5(310)[001]$ GBs for pure metal Mo and U. The grain boundary energy of $\Sigma 3(111)[110]$ of bcc Mo is predicted to be $2.12 \mathrm{~J} / \mathrm{m}^{2}$, while the formation energy of $\Sigma 5(310)$ [001] is predicted to be $1.78 \mathrm{~J} / \mathrm{m}^{2}$, as shown in Table II. The grain boundary properties of Mo were previously studied by DFT, and two values were reported for the $\Sigma 5(310)$ boundary, 
$1.80[20]$ and $1.55 \mathrm{~J} / \mathrm{m}^{2}$ [33], which are close to our results. The grain boundary energies in Mo have also been extensively studied by molecular dynamics simulations [34]. However, these energies are usually lower than those computed by DFT. We have also tested the convergence of the grain boundary energy with respect to the number of layers. For $\Sigma 5(310)[001]$ GB in Mo, doubling the number of layers from 10 to 20 leads to only a $2 \%$ change of the grain boundary energy. Because of the mechanical unstable character of the $\gamma-\mathrm{U}$ phase, studying the GB properties is not straightforward. Without constraint, the GB structure of $\gamma-\mathrm{U}$ can be destroyed in the structural relaxation, and unrealistic negative GB formation energies will be predicted. Therefore only the first few layers of atoms close to grain boundaries were allowed to relax. In this work, the grain boundary energies for $\Sigma 3(111)[110]$ and $\Sigma 5(310)[001]$ GBs in $\gamma$-U are predicted to be 0.13 and 0.52 $\mathrm{J} / \mathrm{m}^{2}$, respectively. We noticed that the grain boundary energies of $\gamma-\mathrm{U}$ are much lower than those of bcc Mo, indicating a weaker bonding in $\gamma$-U than those in bcc Mo. To construct the GBs for the U-Mo alloy phase, we applied the SQS models to the GBs created for bcc metals. Figure 3 shows the created $\Sigma 3(111)[110]$ and $\Sigma 5(310)[001]$ GBs for $\gamma \mathrm{U}$-7Mo. The grain boundary energies in the $\gamma \mathrm{U}-7 \mathrm{Mo}$ alloy are predicted to be 0.37 $\mathrm{J} / \mathrm{m}^{2}$ and $0.63 \mathrm{~J} / \mathrm{m}^{2}$, for $\Sigma 3(111)[110]$ and $\Sigma 5(310)[001] \mathrm{GBs}$, respectively. The grain boundary energies in the U-Mo alloy lie between those of pure $\gamma \mathrm{U}$ and bcc Mo, a result that is consistent with the predicted elastic properties of these systems.

3.2 Grain growth in U-7Mo alloy by phase field modeling 
Reliable physical parameters are critical to a quantitative phase field modeling of grain growth of U-7Mo alloy. However, due to the scarcity of experimental studies of U7Mo alloy, most of these data are not available yet. Therefore, we rely on atomistic simulation methods, such as first-principles methods, to provide such information. We investigated several fundamental thermophysical properties of U-7Mo alloy using firstprinciples method for the first time. The predicted grain boundary energy was utilized to derive the gradient coefficient and chemical potential height for the free energy. Although other properties, such as elastic constants, are not directly used in the current phase field modeling of grain growth, they may be used to study the stress effect on the grain growth in the future.

In real polycrystalline U-7Mo alloy samples, the grain boundary energy $\sigma_{g b}$ and mobility $M$ vary from grain boundary to grain boundary, depending on the grain boundary misorientation and inclination. However, the mathematical models usually ignore the variation of grain boundary energy and mobility. In the present simulations a single value for $\sigma_{g b}$ and $M$ was used for all grain boundaries. This simplified scenario, which is called idea grain growth [35-38], is used extensively by computer simulation.

The input parameters for the phase field simulations are determined as follows. With the grain boundary energies of $\Sigma 3(111)[110]$ and $\Sigma 5(310)[001]$ GBs determined from the DFT calculations in Sec. 3.1, we adopted the relatively smaller value of $\sigma_{g b}=0.37 \mathrm{~J} / \mathrm{m}^{2}(\Sigma 3(111)[110] \mathrm{GB})$ in our phase field simulations, which corresponds to the most stable grain structure. The thickness used in the simulation is assumed to be $4.0 \times 10^{-6} \mathrm{~m}$, which is proximately four grid points at the grain boundary region. Based on the relationship between the grain boundary energy, the gradient coefficient, grain 
boundary thickness $(l)$, and the expansion coefficients of free energy given by Moelans et al [24], $\sigma=\sqrt{2 A \kappa} / 3$ and $l=\sqrt{8 \kappa / A}$ for a symmetrical phase parameter profile where the saddle point of the energy density is located at $\eta_{i}=0.5$, the gradient coefficient $\kappa$ is determined to be $1.11 \times 10^{-6} \mathrm{~J} / \mathrm{m}$ and the expansion coefficients of the free energy are $5.55 \times 10^{5} \mathrm{~J} / \mathrm{m}^{3}, 5.55 \times 10^{5} \mathrm{~J} / \mathrm{m}^{3}$, and $8.33 \times 10^{5} \mathrm{~J} / \mathrm{m}^{3}$ for $A, B$ and $C$, respectively. The activation energy for the GB diffusion of $\mathrm{U}-7 \mathrm{Mo}$ is unavailable in the literature, to our best knowledge. Therefore, for the work reported here, we adopted the activation energy of the GB diffusion measured for Mo [39], that is $2.73 \mathrm{eV}$. We believe this value should be close to that for the U-7Mo alloy, because Mo is the element with much slower diffusivity in U-Mo alloys [40]. The kinetic coefficient $L_{0}$ is related to the GB mobility. Because of the difficulty of calculating this value, we use the experimental data to fit it. In experiments the grain size has been shown to increase from $3 \mu \mathrm{m}$ to $24 \mu \mathrm{m}$ after 0.5 hour (see Table III) at temperature $1000{ }^{\circ} \mathrm{C}$ [7]. Thus, we can derive $L_{0}$ based on the time required for the grain size to increase from $3 \mu \mathrm{m}$ to $24 \mu \mathrm{m}$ in the phase field simulation. $L_{0}$ is determined to be $1.04 \times 10^{-7} \mathrm{~m}^{3} /(\mathrm{Js})$. To give a diffuse interface profile of the domain parameter, we use the function $\phi=0.5-0.5\left(\tanh \frac{R-R_{0}}{\tau}\right)$, where $R_{0}=90 \mu \mathrm{m}$ is the radius of the U-7Mo particle, $\tau=2.0$, and $R$ measures the radius of any grid to the particle center.

In order to study the statistically averaged kinetics and topological features of the ideal two-dimensional grain growth in the phase field model, computer simulations are performed on a simple square lattice by solving Eq. (3). The initial condition of the grain structure in the simulation corresponds to the particle size of $3 \mu \mathrm{m}$, which is consistent with the experimental conditions. The temporal evolution of grain structures at different 
temperatures and with different initial grain size are studied in detail. In order to differentiate the different grains in the simulations, a function $\xi(r)=\sum_{i=1}^{p} \eta_{i}^{2}(r)$ is used, which takes a value of unity within the individual grain and a smaller value along the grain boundary region. Figure 5 shows the grain structures as a function of time with different initial structures heated at a temperature of $1000{ }^{\circ} \mathrm{C}$. The bright regions are grain interiors and the dark lines are grain boundaries. Three different initial grain structures are tested as examples, as shown in Fig. 5: case I with $100 \% 3 \mu \mathrm{m}$ sized grains, case II with $50 \% 3 \mu \mathrm{m}$ sized and 50\% $15 \mu \mathrm{m}$ sized grains, and case II with $75 \% 3 \mu \mathrm{m}$ sized and $25 \% 15 \mu \mathrm{m}$ sized grains. Because of the high annealing temperature, grain boundaries move rapidly, and grains grow in all three cases. The simulation results show that the grain growth is faster with a higher percentage of large grains in the initial structure of the fuel particle. As a comparison, Fig. 6 shows the typical optical micrograph of U-7Mo fuel particles embedded in an $\mathrm{Al}$ matrix that was heat treated at $1000{ }^{\circ} \mathrm{C}$ for half an hour [7]. We want to point out that the circular domain used for the fuel particle can affect the grain shapes at domain boundary. Because of the smooth function of mobility applied on the circular domain, the grain boundaries at the domain boundary do not move, and only the grain boundaries inside the particle evolve. Thus, the grains near the domain boundary evolve differently from the grains inside the particle, which may result in irregular grain shapes at the later stage.

Since the measured grain size data is limited, we have derived a correlation based on the experimental data $[7,41]$ as shown in Table III in order to compare with the current simulation results. The correlation function relates the grain size with the heating time as $d^{2}=d_{0}^{2}+4.63 \times 10^{10} \exp \left(-\frac{32752}{T}\right) t$, where $d_{0}$ is the average grain size in the 
as-fabricated fuel particles (in $\mu \mathrm{m}$ ) and $t$ is the time. The time dependence of the averaged grain size in the fuel particles with three different grain structures, together with the experimentally predicted correlation, is shown in Fig. 7. The average grain size in the particle is estimated by dividing the total area of the fuel particle by the total number of grains. Good agreement between the simulated results and the experimental estimation is obtained when the annealing time is less than 2 hours. Beyond 2 hours, however, a notable deterioration appears. This is due to the small number of grains in the fuel particle at the late stage of annealing, which increases the uncertainty of the estimated grain size. Therefore, calculation of the grain size at the late stage largely depends on the configuration's grain structure. We note that the system with initial 50\% $3 \mu \mathrm{m}$ and 50\% $15 \mu \mathrm{m}$ sized grains evolves faster than the one with $100 \% 3 \mu \mathrm{m}$ sized grains. The reason is that a certain amount of large grains in the system can help the small grain grow faster in the early stage, which makes the average grain size increase faster than the case with $100 \%$ small grains. As shown in Fig. 5, the small grains in case II shrink noticeably faster than those in case I with $100 \% 3 \mu \mathrm{m}$ grains. However, when the system has $75 \% 3 \mu \mathrm{m}$ and $25 \%$ $15 \mu \mathrm{m}$ sized grains as shown in case III, it evolves even faster than case II with $50 \% 3 \mu \mathrm{m}$ and $50 \% 15 \mu \mathrm{m}$ sized grains. We believe this phenomenon is determined by the distribution of grains in the fuel particle. For case III with $25 \%$ large grains, when the large grains are distributed homogeneously in the structure (meaning that large grains are surrounded by abundant small grains), both the small and large grains can grow easily by consuming nearby small grains through the movement of grain boundaries and thus form larger grains. However, for case II with 50\% large grains, the large grains have a high chance to be contacted with nearby large grains. It takes longer time to form larger grains 
by the movement of large grain boundaries than that by small grains. Therefore, the grain growth rate in case II with 50\% large grains is lower than that of case III with 25\% large grains. This result indicates that adding a small amount of large grain in the initial particle can help increase the grain growth rate significantly.

To investigate the effect of temperature on grain growth, we also simulated the evolution of grain structure at a lower temperature of $900{ }^{\circ} \mathrm{C}$. Figure 8 plots the temporal evolutions of grain structures for three different initial grain size distributions. The time dependence of the averaged grain size in the fuel particles with three different grain structures, together with the experimentally predicted correlation, is shown in Fig. 9. The averaged grain size is considerably smaller than that annealed at $1000{ }^{\circ} \mathrm{C}$ for all three cases. The figure shows the time dependence of the average grain size in the fuel particle treated at $900{ }^{\circ} \mathrm{C}$ together with the experimental estimations. The predicted growth rates agree well with the experimental results. The system with $75 \% 3 \mu \mathrm{m}$ and $25 \% 15 \mu \mathrm{m}$ sized grains shows the highest growth rate among the three cases. The case with initial 50\% $3 \mu \mathrm{m}$ and $50 \% 15 \mu \mathrm{m}$ sized grains evolves faster than the $100 \% 3 \mu \mathrm{m}$ grain case. The fact that the average grain size of the case with $50 \% 3 \mu \mathrm{m}$ and $50 \% 15 \mu \mathrm{m}$ initial grains is larger than the other two cases before 1 hour is due to the initial large amount of large grains in the system. These results are consistent with the simulated results at $1000{ }^{\circ} \mathrm{C}$.

Compared with the grain structures treated at $900{ }^{\circ} \mathrm{C}$, the grain size in the fuel particle treated at $1000{ }^{\circ} \mathrm{C}$ is significantly larger. For example, the average grain size increases from $18 \mu \mathrm{m}$ to $43 \mu \mathrm{m}$ after 2 hours when the temperature increased from 900 ${ }^{\circ} \mathrm{C}$ to $1000{ }^{\circ} \mathrm{C}$. Therefore, the annealing time required for the desired grain size can be considerably reduced at higher temperature. The different initial grain size distribution 
also plays an important role in the grain growth rate. By including a small amount of large grain, the grain growth rate can be markedly enhanced for U-Mo fuel particles. However, it is worth noting that the grain growth rate is also affected by the distribution of grains in the fuel particle. Therefore, it is difficult to quantify an optimal amount of large grains which leads to the largest grain growth rate.

\section{Conclusions}

Using a multiscale simulation approach, we investigated the grain growth in U7Mo alloy for nuclear fuel application. DFT calculations were used to study the mechanical stability of $\gamma \mathrm{U}-7 \mathrm{Mo}$ alloy based on the predicted elastic properties. Two grain boundaries, $\Sigma 3(111)[110]$ and $\Sigma 5(310)[001]$, were investigated for $\gamma$ U-7Mo and compared with those of pure bcc Mo and U metals. Using the predicted grain boundary properties as input parameter, we studied the effects of annealing temperature, annealing time and initial grain structure on the grain growth in U-7Mo alloy particles by the phase field approach. Increasing the heating temperature is found to effectively reduce the annealing time required for the desired grain size. We found that the fuel particle with a certain amount of mixed sized grains has a faster grain growth rate than those with identical sized grains. The predicted grain growth rate is in good agreement with the empirical correlation derived from experimental measurements. We expect that the currently predicted kinetic model for the grain growth in the U-7Mo will be helpful in determining the optimal experimental conditions for annealing. 


\section{Acknowledgments}

This work is sponsored by the U.S. Department of Energy, National Nuclear Security Administration (NNSA), Office of Material Management and Minimization (NA-23) Reactor Conversion Program. Use of the Center for Nanoscale Materials, an Office of Science user facility, was supported by the U. S. Department of Energy, Office of Science, Office of Basic Energy Sciences, under Contract No. DE-AC02-06CH11357. We gratefully acknowledge the computing resources provided on Blues, a highperformance computing cluster operated by the Laboratory Computing Resource Center at Argonne National Laboratory. 


\section{References}

[1] Y.S. Kim, G.L. Hofman, M.R. Finlay, J.L. Snelgrove, S.L. Hayes, M.K. Meyer, C.R. Clark, F. Huet, . the 25th International Meeting on Reduced Enrichment for Research and Test Reactors (RERTR). Chicago, Illinois, 2003.

[2] J. Rest, G.L. Hofman, Y.S. Kim, Journal of Nuclear Materials 385 (2009) 563.

[3] J. Rest, Journal of Nuclear Materials 346 (2005) 226.

[4] Y.S. Kim, G.L. Hofman, J.S. Cheon, Journal of Nuclear Materials 436 (2013) 14.

[5] Y.S. Kim, J.M. Park, K.H. Lee, B.O. Yoo, H.J. Ryu, B. Ye, Journal of Nuclear Materials 454 (2014) 238.

[6] J.M. Park, Y.S. Han, K.H. Kim, Y.S. Lee, C.K. Kim. Fabrication and characterization of atomized U-Mo powder dispersed fuel compacts for the RERTR-3 irradiation test. the 25th International Meeting on Reduced Enrichment for Research and Test Reactors (RERTR). Budapest, Hungary, 1999.

[7] T.C. Wiencek, E. O'Hare. 2015 (unpublished work).

[8] L.Q. Chen, Annual Review of Materials Research 32 (2002) 113.

[9] I. Steinbach, Annual Review of Materials Research, Vol 4343 (2013) 89.

[10] L. Bellaiche, D. Vanderbilt, Physical Review B 61 (2000) 7877.

[11] A. Zunger, S.H. Wei, L.G. Ferreira, J.E. Bernard, Physical Review Letters 65 (1990) 353.

[12] A. Landa, P. Söderlind, P.E.A. Turchi, L. Vitos, A. Ruban, Journal of Nuclear Materials 385 (2009) 68.

[13] A. Landa, P. Söderlind, P.E.A. Turchi, Journal of Nuclear Materials 414 (2011) 132. 
[14] A. van de Walle, G. Ceder, Journal of Phase Equilibria 23 (2002) 348.

[15] A. van de Walle, P. Tiwary, M. de Jong, D.L. Olmsted, M. Asta, A. Dick, D. Shin, Y. Wang, L.Q. Chen, Z.K. Liu, Calphad 42 (2013) 13.

[16] P.E. Blöchl, Physical Review B 50 (1994) 17953.

[17] G. Kresse, J. Furthmüller, Phys. Rev. B 54 (1996) 11169.

[18] G. Kresse, D. Joubert, Phys. Rev. B 59 (1999) 1758.

[19] J.P. Perdew, K. Burke, M. Ernzerhof, Phys. Rev. Lett. 77 (1996) 3865.

[20] T. Ochs, C. Elsässer, M. Mrovec, V. Vitek, J. Belak, J.A. Moriarty, Philosophical Magazine A 80 (2000) 2405.

[21] H. Ogawa, MATERIALS TRANSACTIONS 47 (2006) 2706.

[22] D. Fan, L.Q. Chen, Acta Materialia 45 (1997) 611.

[23] D.N. Fan, C.W. Geng, L.Q. Chen, Acta Materialia 45 (1997) 1115.

[24] N. Moelans, B. Blanpain, P. Wollants, Physical Review B 78 (2008).

[25] L.Y. Liang, M. Stan, M. Anitescu, Applied Physics Letters 105 (2014).

[26] M. Wang, B.Y. Zong, G. Wang, Computational Materials Science 45 (2009) 217.

[27] L.Q. Chen, J. Shen, Computer Physics Communications 108 (1998) 147.

[28] S. Shang, Y. Wang, Z.-K. Liu, Applied Physics Letters 90 (2007) 101909.

[29] D.H. Chung, W.R. Buessem, Journal of Applied Physics 38 (1967) 2535.

[30] J.M. Dickinson, P.E. Armstrong, Journal of Applied Physics 38 (1967) 602.

[31] B. Beeler, C. Deo, M. Baskes, M. Okuniewski, Journal of Nuclear Materials 433 (2013) 143. 
[32] F. Tasnádi, M. Odén, I.A. Abrikosov, Physical Review B 85 (2012) 144112.

[33] A.M. Tahir, R. Janisch, A. Hartmaier, Modelling and Simulation in Materials Science and Engineering 21 (2013) 075005.

[34] S. Ratanaphan, D.L. Olmsted, V.V. Bulatov, E.A. Holm, A.D. Rollett, G.S. Rohrer, Acta Materialia 88 (2015) 346.

[35] N. Moelans, F. Wendler, B. Nestler, Computational Materials Science 46 (2009) 479.

[36] C.E. Krill, L.Q. Chen, Acta Materialia 50 (2002) 3057.

[37] R.D. Kamachali, I. Steinbach, Acta Materialia 60 (2012) 2719.

[38] S.G. Kim, D.I. Kim, W.T. Kim, Y.B. Park, Physical Review E 74 (2006).

[39] D.C. Blaine, J.D. Gurosik, S.J. Park, D.F. Heaney, R.M. German, Metallurgical and Materials Transactions a-Physical Metallurgy and Materials Science 37A (2006) 715.

[40] D.E. Smirnova, A.Y. Kuksin, S.V. Starikov, Journal of Nuclear Materials 458 (2015) 304.

[41] J.M. Park. 2015 (unpublished work). 
Table I. Calculated elastic properties of bcc Mo, $\gamma-\mathrm{U}$ and $\gamma \mathrm{U}-7 \mathrm{Mo}$, including elastic constants $\mathrm{C}_{\mathrm{ij}}(\mathrm{GPa})$, shear constant $(\mathrm{GPa})$, bulk modulus (GPa), shear modulus $(\mathrm{GPa})$, Young's modulus (GPa), and Poisson ratio.

\begin{tabular}{lccccccccc}
\hline & $C_{11}$ & $C_{12}$ & $C_{44}$ & $C$ & $B$ & $G$ & $E$ & $v$ & Reference \\
\hline \multirow{2}{*}{ Bcc Mo } & 466 & 157 & 103 & 155 & 260 & 122 & 316 & 0.298 & This work \\
& 464 & 158 & 109 & 153 & 260 & 125 & 323 & 0.293 & Expt. [30] \\
& 94 & 154 & 34 & -30 & 134 & 114 & 225 & 0.169 & This work \\
$\gamma-\mathrm{U}$ & 86 & 155 & 37 & -35 & 132 & 113 & 265 & 0.17 & Calc. [31] \\
& 173 & 138 & 50 & 23 & 143 & 36 & 100 & 0.383 & This work \\
\hline U-7Mo & 173 & & & & & & & \\
\hline
\end{tabular}


Table II. Grain boundary energy (eV) in bcc Mo, $\gamma-\mathrm{U}$ and $\gamma \mathrm{U}$-7Mo calculated by DFT.

\begin{tabular}{llll}
\hline & $\Sigma 3(111)[110]$ & $\Sigma 5(310)[100]$ & Reference \\
\hline Bcc Mo & 2.12 & 1.78 & This work \\
& & $1.70[20], 1.52[33]$ & Calc. \\
$\gamma-\mathrm{U}$ & 0.13 & 0.52 & This work \\
$\mathrm{U}-7 \mathrm{Mo}$ & 0.37 & 0.63 & This work \\
\hline
\end{tabular}


Table III. U-Mo powder annealing test results from previous experimental studies.

\begin{tabular}{cccc}
\hline & Temperature $\left({ }^{\circ} \mathrm{C}\right)$ & Time $(\mathrm{s})$ & Grain size $(\mu \mathrm{m})$ \\
\hline ANL RERTR-3 [6] & 800 & 360000 & 22 \\
ANL [7] & 900 & 3600 & 23 \\
KAERI [41] & 1000 & 3600 & 20 \\
ANL [7] & 1000 & 1800 & 24 \\
\hline
\end{tabular}




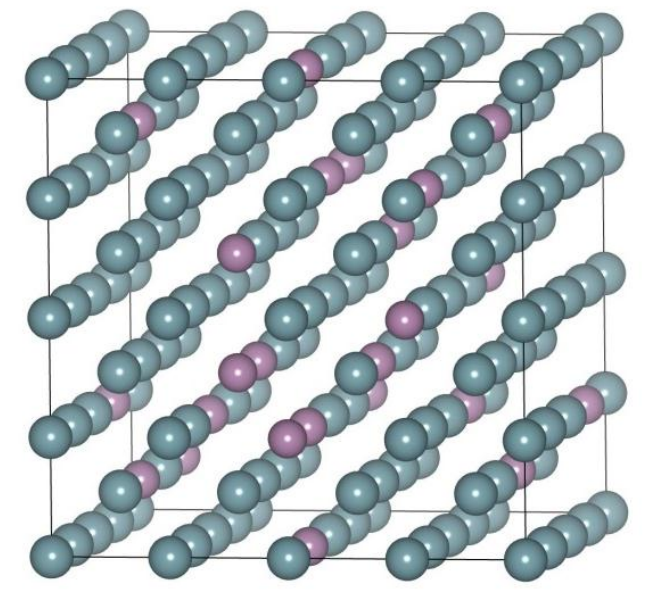

Figure 1. SQS model of U-7Mo alloy with 128 atoms. 
(a)

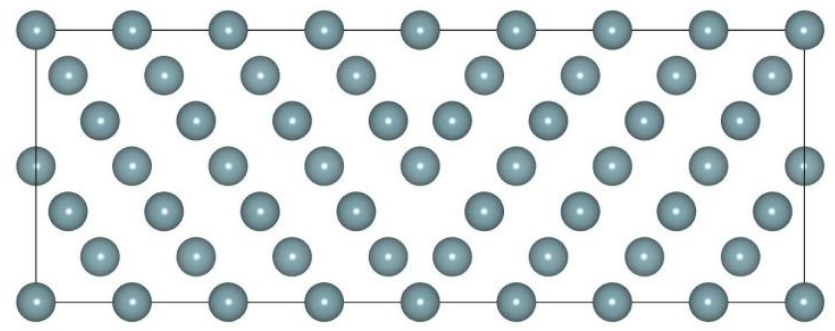

(b)

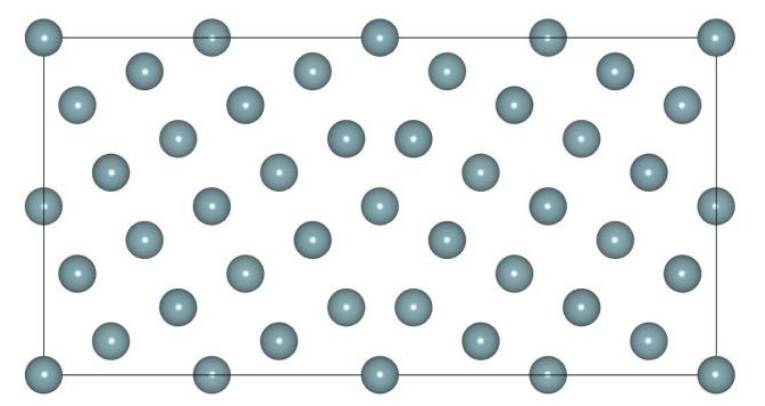

Figure 2. Atomic structures of (a) $\Sigma 3(111)[110]$ and (b) $\Sigma 5(310)[001]$ STGBs for bcc metals, such as, Mo and U. 
(a)

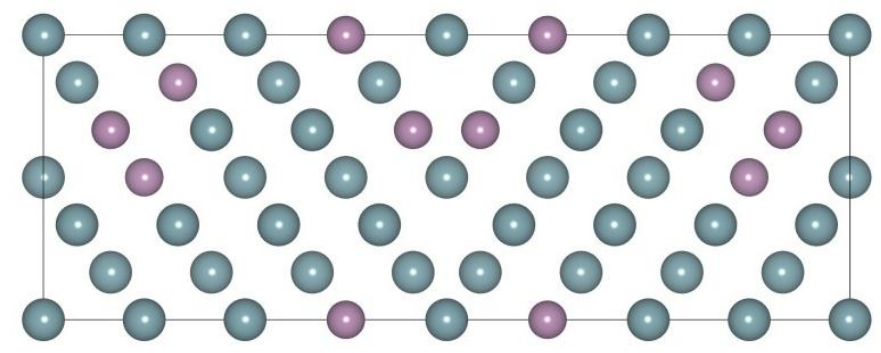

(b)

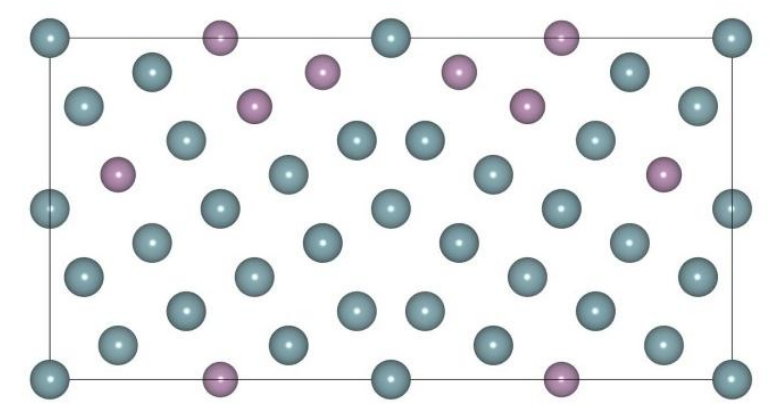

Figure 3. Atomic structures of (a) $\Sigma 3(111)[110]$ and (b) $\Sigma 5(310)[001]$ STGBs for $\gamma$ U7Mo alloy. Blue and purple atoms represent the $\mathrm{U}$ and Mo atoms, respectively. 


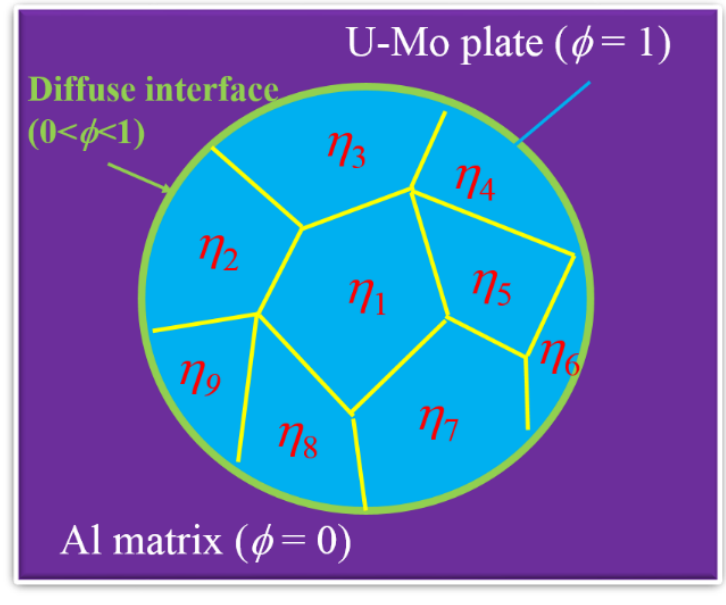

(a)

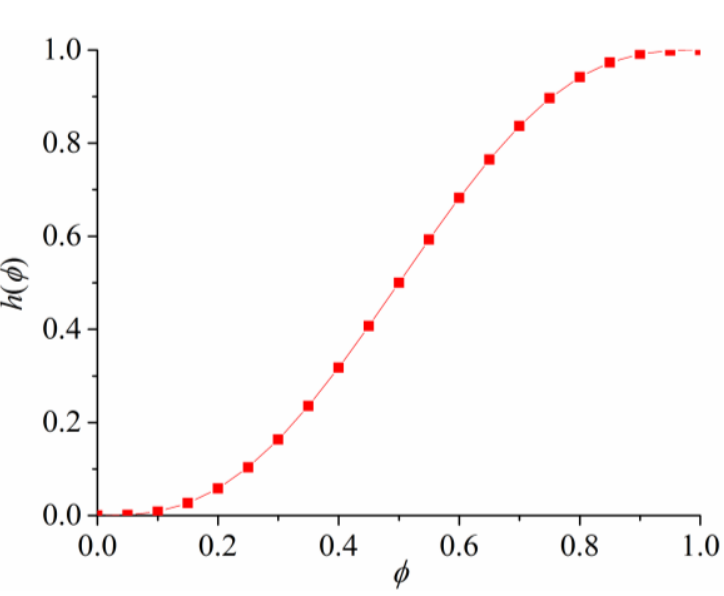

(b)

Figure 4. (a) Schematic diagram of intercalated a U-7Mo fuel particle with different grains embedded in Al matrix. (b) $h$ function vs domain parameter. 
Case I:

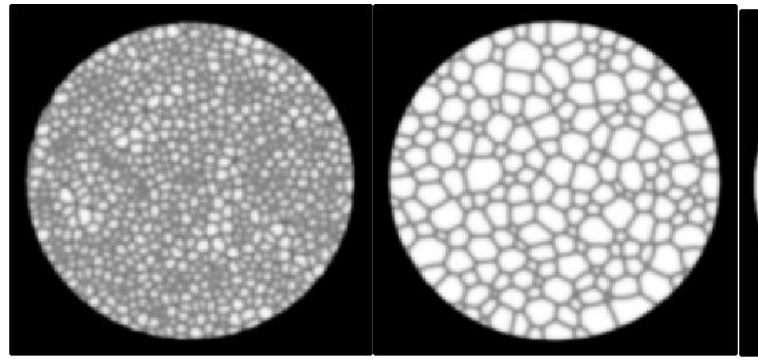

(a) $0 \mathrm{~h}$;

(b) $0.083 \mathrm{~h}$;

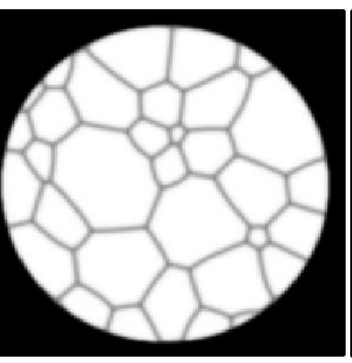

(c) $0.83 \mathrm{~h}$;

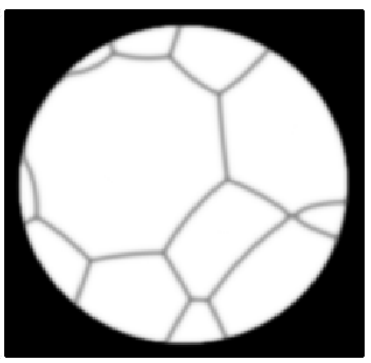

(d) $3.33 \mathrm{~h}$.

Case II:

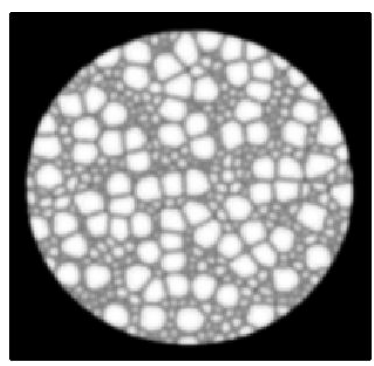

(a) $0 \mathrm{~h}$;

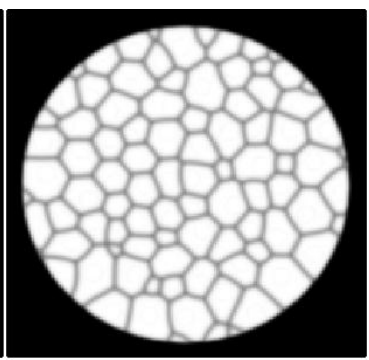

(b) $0.083 \mathrm{~h}$;

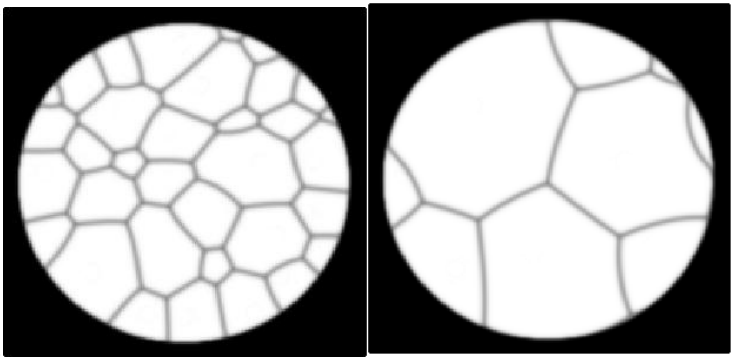

(c) $0.83 \mathrm{~h}$;

(d) $3.33 \mathrm{~h}$.

Case III:

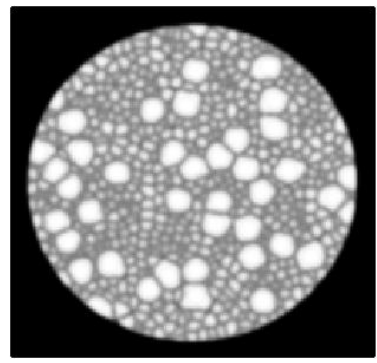

(a) $0 \mathrm{~h}$;

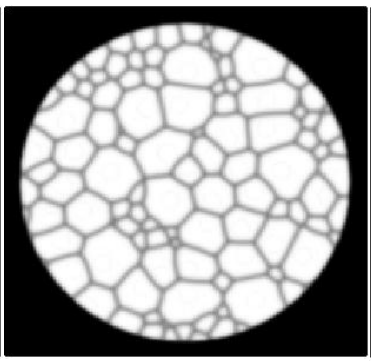

(b) $0.083 \mathrm{~h}$;

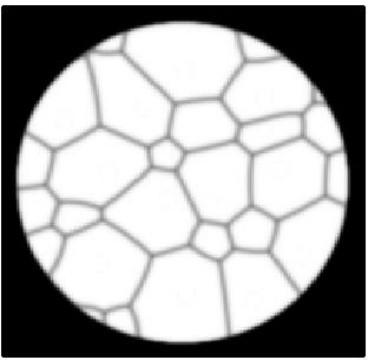

(c) $0.83 \mathrm{~h}$;

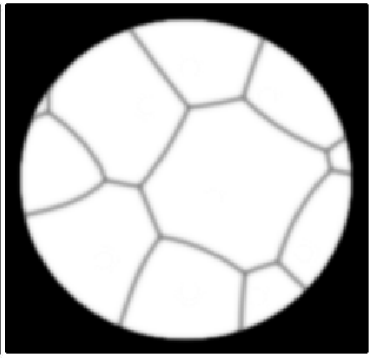

(d) $3.33 \mathrm{~h}$.

Figure 5. Temporal evolution of the grain structures in a U-7Mo particle at $1000{ }^{\circ} \mathrm{C}$ : (a) 0 h; (b) $0.083 \mathrm{~h}$; (c) $0.83 \mathrm{~h}$; and (d) $3.33 \mathrm{~h}$. Case I: The initial grain size in the fuel particle 
is $100 \% 3 \mu \mathrm{m}$. Case II: The initial grain size in the fuel particle is $50 \% 3 \mu \mathrm{m}$ and $50 \%$ $15 \mu \mathrm{m}$. Case III: The initial grain size in the fuel particle is $75 \% 3 \mu \mathrm{m}$ and $25 \% 15 \mu \mathrm{m}$. 


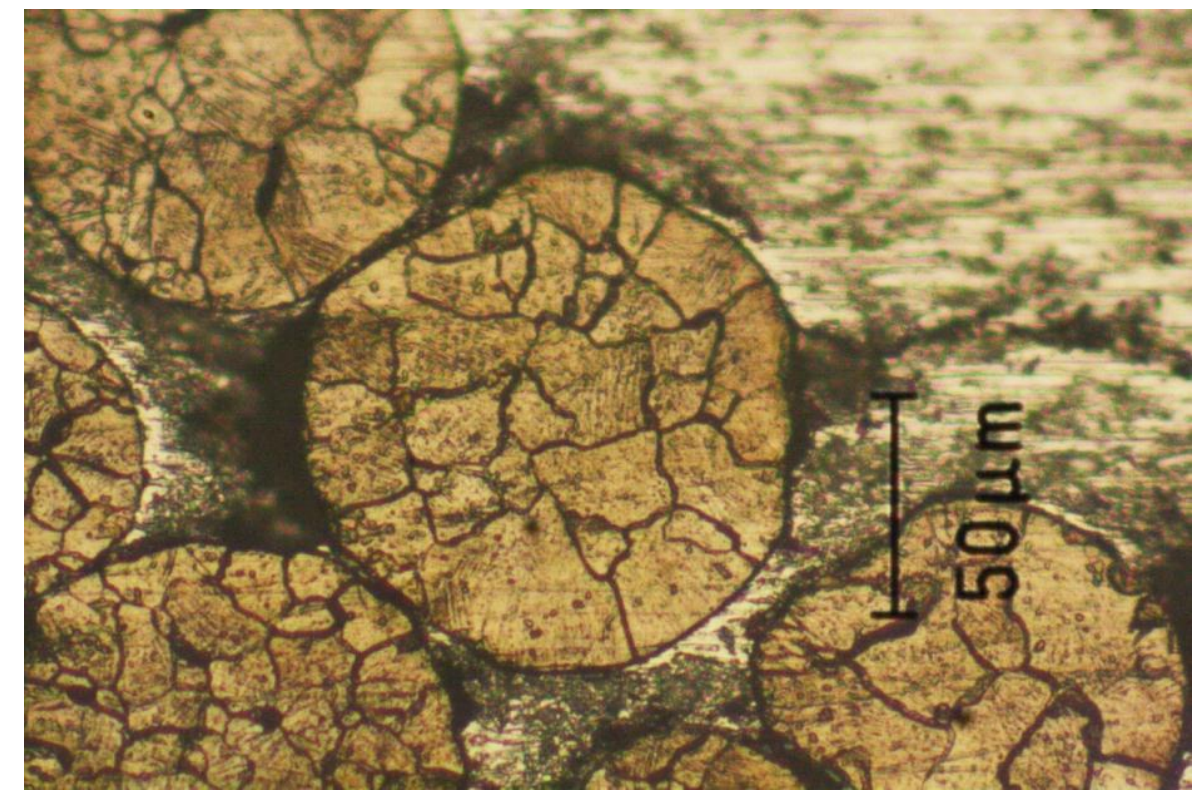

Figure 6. Optical micrograph image of $\mathrm{ZrN}$ coated $\mathrm{U}-7 \mathrm{Mo}$ fuel particles embedded in $\mathrm{Al}$ matrix after heat treatment at $1000{ }^{\circ} \mathrm{C}$ for half an hour. 


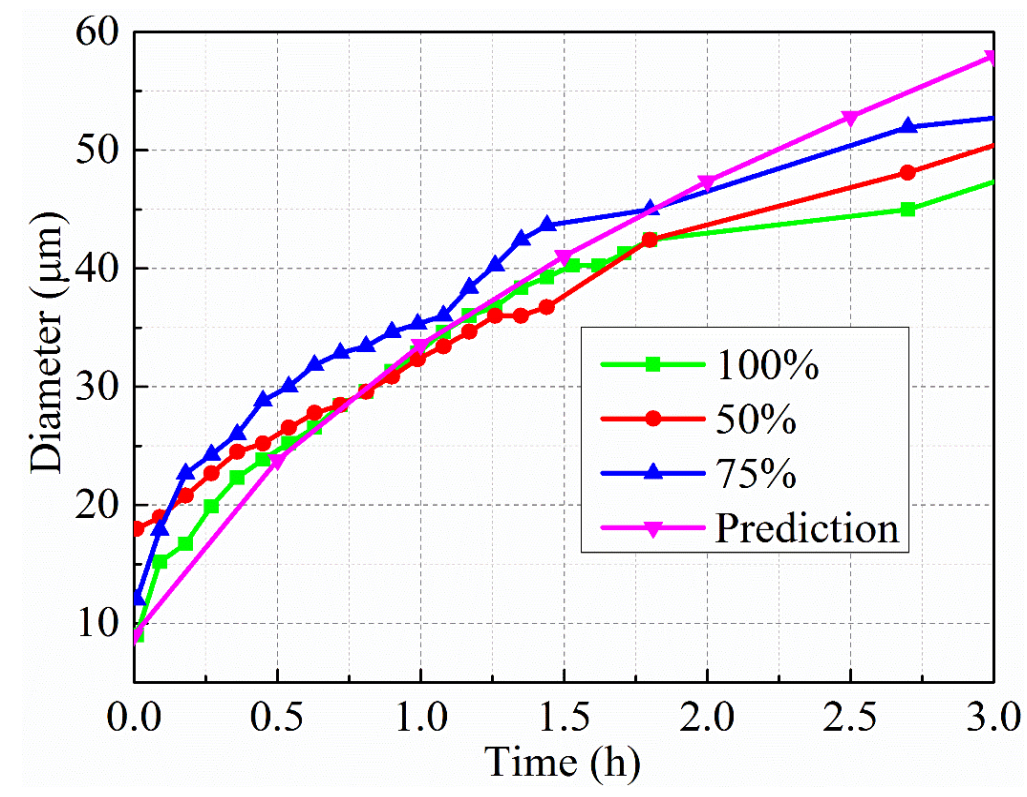

Figure 7. Average grain size in a U-7Mo particle vs time at $1000{ }^{\circ} \mathrm{C}$ with an initial grain

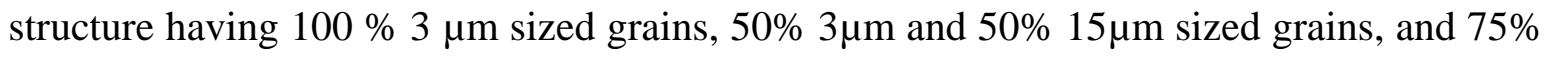
$3 \mu \mathrm{m}$ and $25 \% 15 \mu \mathrm{m}$ sized grains, respectively. The experimentally predicted grain size is also plotted. 
Case I:

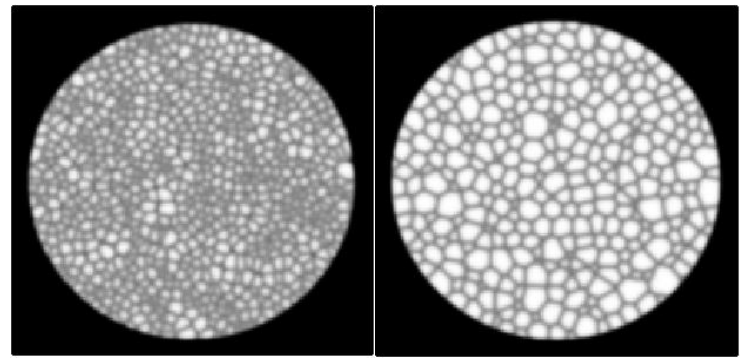

(a) $0 \mathrm{~h}$;

(b) $4.1 \mathrm{~h}$;

Case II.

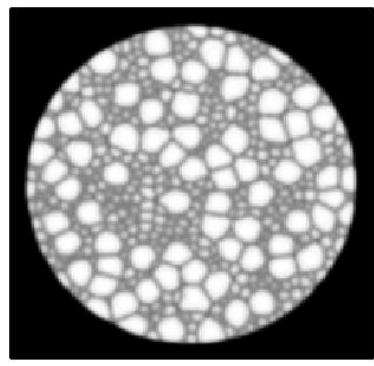

(a) $0 \mathrm{~h}$;

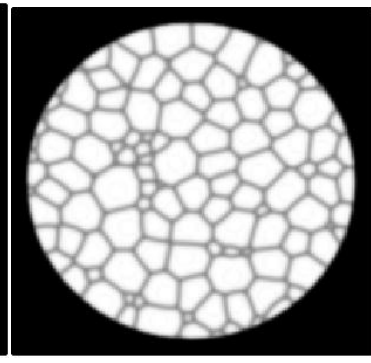

(b) $4.1 \mathrm{~h}$;

Case III.

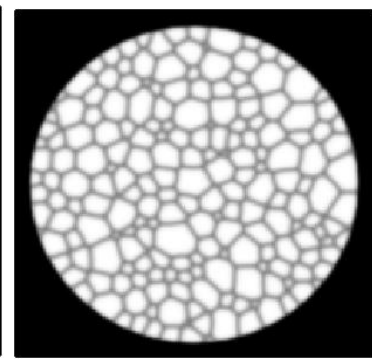

(c) $8.1 \mathrm{~h}$;

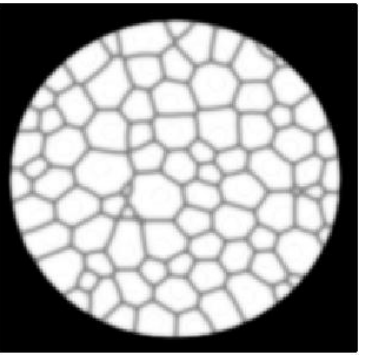

(c) $8.1 \mathrm{~h}$;

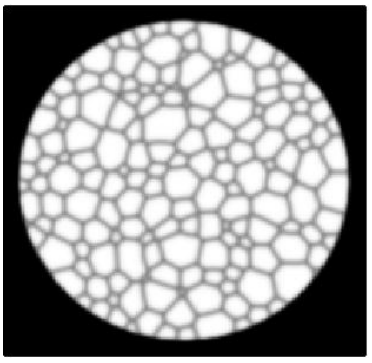

(d) $10.5 \mathrm{~h}$.

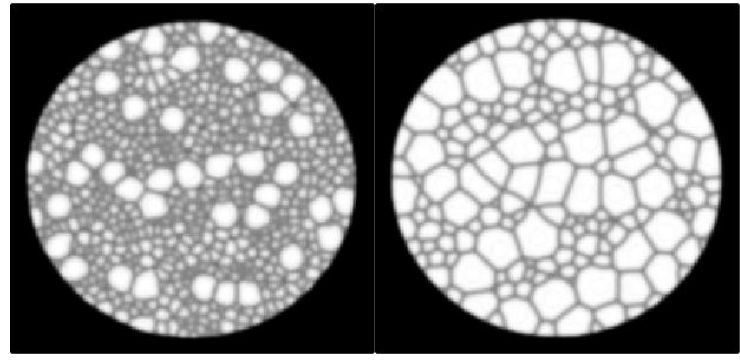

(a) $0 \mathrm{~h}$;

(b) $4.1 \mathrm{~h}$;

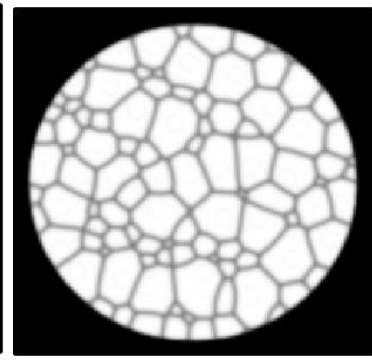

(c) $8.1 \mathrm{~h}$; (d) $10.5 \mathrm{~h}$.

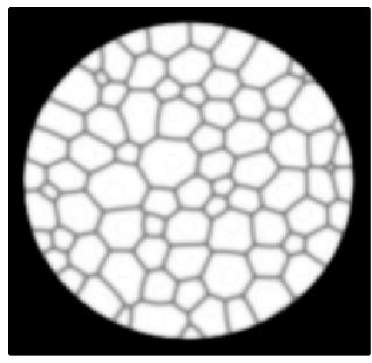

Figure 8. Temporal evolution of the grain structures in a U-7Mo particle at $900{ }^{\circ} \mathrm{C}$ : (a) 0 $\mathrm{h}$; (b) $4.1 \mathrm{~h}$; (c) $8.1 \mathrm{~h}$; and (d) $10.5 \mathrm{~h}$. Case I: The initial grain size in the fuel particle is $100 \% 3 \mu \mathrm{m}$. Case II: The initial grain size in the fuel particle is $50 \% 3 \mu \mathrm{m}$ and $50 \% 15 \mu \mathrm{m}$. Case III: The initial grain size in the fuel particle is $75 \% 3 \mu \mathrm{m}$ and $25 \% 15 \mu \mathrm{m}$. 


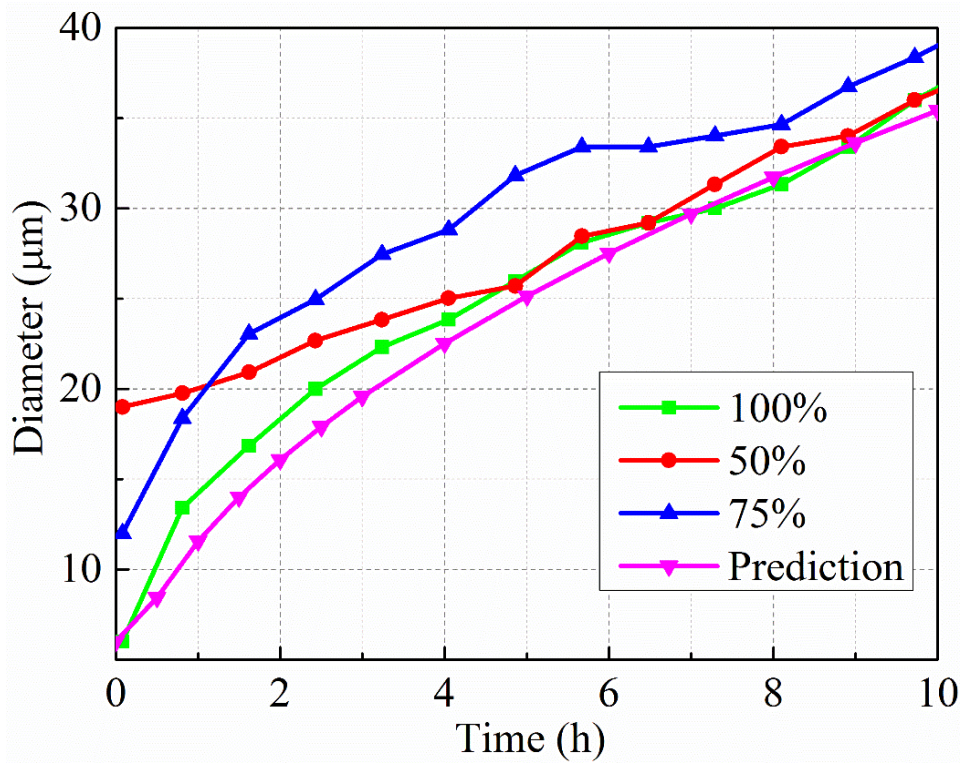

Figure 9. Average grain size in a U-7Mo particle vs time at $900{ }^{\circ} \mathrm{C}$ with an initial grain structure having $100 \% 3 \mu \mathrm{m}$ sized grains, 50\% $3 \mu \mathrm{m}$ and 50\% $15 \mu \mathrm{m}$ sized grains, and $75 \%$ $3 \mu \mathrm{m}$ and $25 \% 15 \mu \mathrm{m}$ sized grains, respectively. The experimentally predicted grain size is also plotted. 